\title{
Molecular basis of lysosomal storage disorders in India
}

\author{
Shweta P Kondurkar ${ }^{1 *}$, Parag Tamhankar ${ }^{1}$, Pratima Kondurkarr ${ }^{1}$, Ashwin Dalal ${ }^{2}$, Jayesh Sheth ${ }^{3}$ \\ From International Conference on Human Genetics and 39th Annual Meeting of the Indian Society of \\ Human Genetics (ISHG) \\ Ahmadabad, India. 23-25 January 2013
}

\section{Background}

Lysosomal Storage Diseases(LSD) are a group of rare recessive inherited metabolic disorders that result from the deficiency of a single enzyme required for the metabolism of lipids, glycoproteins or mucopolysaccharides. There is a lack of data on molecular basis of LSDs in India . The current study involves molecular analysis of patients with Tay Sachs disease(TSD) (HEXA gene), Sandhoff disease(SD) (HEXB gene), Gaucher disease (GD) (GBA gene), GM1 gangliosidosis (GG) (GLB1 gene), metachromatic leukodystrophy (MLD) (ARSA gene), and Pompe disease (PD) (GAA gene).

\section{Materials and methods}

Patients presenting during the year 2011-2013 with characteristic clinical features of the above LSDs underwent specific biochemical testing (leucocyte enzyme assay) followed by sequencing of the respective gene. Enzyme assays performed include total hexosaminidase and hexosaminidase B (TSD and SD), glucocerebrosidase (GD), beta galactosidase (GG), arylsulphatase A (MLD) and alpha glucosidase (PD). The molecular basis of disease in patients with enzyme deficiency was confirmed by bidirectional Sanger sequencing covering all the exons and exon-intron boundaries of the respective genes.

\section{Results}

During the study period, 131 unrelated families across India were studied. These included 47 families of TSD, 36 families with SD, 15 families with GD, 8 families with GG, 15 families with MLD, and 10 families with PD. Ninety two families (70.2 \%) families showed consanguinity. The age range for patients was between 3 months to 7 years; however, one patient with PD was adult (21 years). The youngest patient had PD. The study identified 218 mutant alleles in 119 patients. Only ten alleles were recurrent. Founder mutation was identified in TSD patients from Gujarat (p.E462V) which will help screening in patients from this state. Also in SD, the mutation hotspot R284X represented $23.3 \%$ of alleles (7/30). No mutations could be identified in 12 patients and the second mutation could not be identified in 10 patients, despite being biochemically confirmed.

\section{Conclusion}

The study shows allelic heterogeneity in Indian patients with LSDs. A molecular screening strategy for the common mutations could be adopted for TSD and SD patients.

\section{Authors' details}

'ICMR Genetic Research Centre (NIRRH), Mumbai, India. ${ }^{2}$ Center for DNA Fingerprinting and Diagnostics, Hyderabad, India. ${ }^{3}$ Foundation for Research in Genetics and Endocrinology, Ahmedabad, Gujarat, India.

Published: 21 January 2014

doi:10.1186/1755-8166-7-S1-P130

Cite this article as: Kondurkar et al:: Molecular basis of lysosomal storage disorders in India. Molecular Cytogenetics 2014 7(Suppl 1):P130.

* Correspondence: shwetaprakashkondurkar@yahoo.com

${ }^{1}$ ICMR Genetic Research Centre (NIRRH), Mumbai, India

Full list of author information is available at the end of the article 